\title{
Epilepsy in Multigene Tubulin Family Mutations
}

Romina Romaniello and Renato Borgatti*

Neuropsychiatry and Neurorehabilitation Unit, Scientific Institute, IRCCS Eugenio Medea, Bosisio Parini, Lecco, Italy

*Corresponding author: Renato Borgatti, Neuropsychiatry and Neurorehabilitation Unit, Scientific Institute, IRCCS Eugenio Medea, Via D. L. Monza 20, 23842 Bosisio Parini, Lecco, Italy. Tel: 0039031877111 , Fax 0039 031877499; E-mail: renato.borgatti@bp.lnf.it

Received date: October 14, 2015; Accepted date: November 18, 2015; Published date: November 25, 2015

Copyright: (2015 Borgatti R, et al. This is an open-access article distributed under the terms of the Creative Commons Attribution License, which permits unrestricted use, distribution, and reproduction in any medium, provided the original author and source are credited.

\section{Abstract}

Mutations in $\alpha$ - and $\beta$-tubulin genes are responsible for a large spectrum of brain malformations secondary to abnormal neuronal migration, organization, differentiation, and axon guidance and maintenance. Motor and language developmental disorders, cognitive impairment and epilepsy are the main clinical associated symptoms. Frequency and severity of these disorders are largely related with the involvement of specific tubulin genes and their functions.
\end{abstract}

The present study summarizes all the published data on tubulin family gene mutations and the associated clinical phenotype in order to define epilepsy recurrence and its characteristics.

Mutations disrupting the stability of microtubules, mainly mechanisms involving neuronal migration and organization, may play an important role in epileptogenicity. Moreover, since mutations in the $\alpha-$ and $\beta$ - tubulin genes are responsible for a large spectrum of cortical and brain malformations, they do not show a high specificity and correlation with an epileptic phenotype unlike other MCDs-related genes. Usually seizures start early (in the first year of life) and they are more often generalized (infantile spasms, tonic, tonic-clonic) as expression of a diffuse cortical maldevelopment.

Mutations in the TUBA1A gene, causing various degrees of agyria-pachigyria spectrum, classical lissencephaly or polymicrogyria, represent among tubulin genes, the major responsible of epilepsy (about $50 \%$ of the cases).

Disorders of cortical development as polymicrogyria, various types of gyral disorganization and schizencephaly were also described in cases carrying TUBB2B gene mutations showing epilepsy in the $40 \%$.

As far as mutations in the TUBB4A gene are concerned, despite the brain phenotype is represented by a form of leukoencephalopathy characterized by hypomielination with atrophy of the basal ganglia and cerebellum and not by MCDs, epilepsy is present in about $40 \%$ of the mutated subjects.

On the contrary, mutations in the TUBB3 gene cause epilepsy only in $15 \%$ of cases, while seizures have never been described in association with TUBB gene.

Keywords: Tubulin Genes; Epilepsy; MCDs; Axonal guidance; TUBA1A; TUBB2B; TUBB3

\section{Introduction}

Epilepsy, one of the most common neurological disorders, may present multiple etiologies.

Over the years, studies on families with multiple affected individuals have provided evidence about the key role of genes in determining epilepsy [1-3]. Two main different groups of genes have been studied: first, mutations in genes playing a role in neuronal membrane electrical stabilization (mostly channellopathy), not associated with structural cortical abnormalities [1]; second, mutations in genes regulating the brain development leading to major structural cortical abnormalities (malformations of cortical development, MCDs) associated with specific neuroradiological patterns $[2,3]$. In these last cases, the involved genes govern mechanisms of neuronal proliferation, migration and postmigrational cortical organization and connectivity.
About two decades ago, with the widespread availability of new molecular genetics and neuroradiology techniques, the study of the genes involved in processes of cerebral cortical development has greatly increased, and more than 100 genes have been reported to date [2,4,5]. Among MCDs-related genes, a key role is played by genes encoding microtubules (MTs), proteins with the function of providing structures and forces needed by neurons to migrate and to develop axonal and dendritic processes [6]. In recent years, mutations in the tubulin multigene family have been suggested to be involved in the alteration of the dynamic properties and functions of microtubules in several ways, leading to a broad spectrum of disorders of brain development, namely "Tubunopathies" $[4,7,8]$.

The present review describes in detail the tubulin genes family and its role in brain development. All the published data on tubulin family gene mutations and the associated clinical phenotype are summarized in order to define epilepsy recurrence and its characteristics. 


\section{Multigene tubulin family}

In higher eukaryotes, tubulin proteins are encoded by the family of neuronally expressed $\alpha$ - and $\beta$ - tubulin genes (i.e., TUBA1A, TUBB2B, TUBB3, TUBB4A, TUBB2A, TUBB, TUB8A), evolutionary conserved among different species, mainly expressed in post mitotic neurons in the early stage of cortical development, with a specific spatial and temporal expression pattern. Tubulin proteins play a key role in several cellular processes crucial for a proper cortical development during neuronal proliferation, migration, differentiation, cortical laminar organization, and axonal guidance of the radial glia (axon outgrowth and maintenance) [7].

Tubulins are dimeric proteins consisting of two closely $\alpha$ and $\beta$ related subunits and represent the major constituents of microtubules [9]. Each tubulin protein contains three separate structural domains (i.e., $\mathrm{N}$-terminal nucleotide-binding, intermediate, $\mathrm{C}$-terminal). The $\mathrm{N}$ Terminal nucleotide-binding domain forms the GTP binding site required for protein folding and stability and the proper conformation of longitudinal protofilaments. This highly conserved region is found in both chains and acts as a flexible loop controlling the access of the nucleotide to the binding site. Its interactions with the intermediate domain leads to structural rearrangements, resulting from the hydrolysis of the GTP bound and microtubule depolymerization. Other residues within this domain mediate longitudinal and lateral interactions, flanking the side and inner surface on heterodimer that are necessary for both heterodimer and microtubule stability. Residues at the C-terminus, forming a helices on the external surface of the tubulin, interact with motor proteins and microtubule-associated proteins (MAPs) kinesin and dynein, regulating the dynamic behavior of microtubules, necessary for neuronal migration, differentiation and axonal guidance [9-11].

The microtubule has highly specialized roles within neurons during brain development. The disruption of microtubule-based processes can lead to impairment in neuronal migration and axon tract formation, leading to a broad range of brain malformations. Therefore, mechanisms of microtubule stability are involved in neuronal migration, while microtubule dynamic in the growth of axonal cone is required for axonal growth [11-14].

Mutations in the $\alpha$ - and $\beta$ - tubulin genes may be widely distributed among the three domains of the proteins, perturbing dynamic properties and functions of microtubules according to their structural location. Mutations can disrupt interaction with the GTP nucleotide or the region adjacent to it, which is relevant to the interaction and regulation action of microtubule dynamics. Another subset of mutations can be located at the contact surfaces between the intra and the heterodimer leading to alteration of the longitudinal protofilament interactions [11-14]. A third group of mutations disrupt residues laying in proximity to the interface between the $\mathrm{N}$-terminal and the intermediate domains, affecting the heterodimer stability and/or the dynamic properties of microtubules, with severe implications in processes of neuronal migration [8]. Finally, mutations can interact with residues on the surface of microtubules, that mediate protein interactions with kinesin, dynein and other MAPs, with consequences on axonal guidance and maintenance.

Mutations in $\alpha$ - and $\beta$ - tubulin genes are responsible for a large spectrum of overlapping brain malformations characterized by abnormal neuronal migration, organization, differentiation, and axon guidance and maintenance as cortical abnormalities (agyria-pachigyria spectrum, nodular heterotopias, polymicrogyria and schizencephaly), defects of commissural axon tracts, dysmorphisms of basal ganglia and brainstem, rare forms of leucoencephalopathy with degeneration of motor and sensor axons and, as recently described, cerebellar dysplasia [10,11,15-23]. Despite this overlapping phenotype, mutation-specific phenotypes in tubulin isotypes exist, due both to the specific function of the related isotype and to the different functional areas of the protein involved leading to a genotype/phenotype correlation, based not only on the primary sequence of the protein, but also on the tertiary structure and the three-dimensional arrangement of the protein [9].

In a recent study, Cushion et al analyzed indeed the correlation between $\alpha$ - and $\beta$ - tubulin genes mutation location and the associated broad phenotype, showing how variability in tubulin-related disorders depends on how each aminoacid substitution alters specific microtubule functions (i.e., mutations that predominantly interfere with heterodimerization may not affect cortical development) [15].

\section{Tubulin clinical phenotypes and epileptogenesis}

The role of mutations in the $\alpha$ - and $\beta$-tubulin genes family in the determinism of epilepsy is complex, depending on the way they alter the dynamic properties and functions of microtubules. This may include: the reduction of the number of functional tubulin heterodimers, the alteration of GTP binding and the disruption of the binding of other proteins to microtubules (motor proteins and other MAPs). Among them, the main role in determining epilepsy is played by mutations that disrupt stability of MTs, which are primarily involved in mechanisms of neuronal migration and organization, while perturbations of the microtubule dynamic, which have a crucial function in the axonal growth and guidance, are less frequently associated with epileptic phenotypes.

As widely underlined by recent literature, the key features related to tubulin-genes disorders are expression of abnormal axonal guidance mechanisms, such as defects of commissural axon tracts and dysmorphisms of basal ganglia, rather than cortical abnormalities expression of abnormal neuronal migration mechanisms [10,13,24-26]. Consequently, in tubulin-genes disorders, epilepsy should not be considered a predominant symptom. When present, it is described with a wide range of severity (from a mild clinical presentation and spontaneous regression to intractable seizures, such as infantile spasms), without a specific pattern and it is often associated with MCDs [27].

In fact, malformations of cortical development are the major cause of epilepsy with a range of severity depending on the type of cortical defects. The seizures onset typically starts early in infancy or childhood. Epilepsy in MCDs is the consequence of either the malpositioning of normal cortical neurons or the presence of abnormal cortical neurons leading to abnormal cortical circuitries. An imbalance between the excitatory (glutaminergic) and inhibitory (Gammaaminobutyric acid, GABAergic) systems, which would normally control electrical discharges and prevent spontaneous abnormal electrical discharges and seizures, may be consequently observed. Dysmaturity of neuronal networks and GABA synaptic activity cause depolarizing actions similar to immature developing networks, thus activating epileptogenic circuitry [5,28]. Moreover, since they have a key role in several pathways of cortical development, mutations in genes encoding microtubule-related proteins might result in epileptic disorders, as the consequence of failure of normal neural circuit formation. 
On the basis of the type of MCDs, specific clinical patterns can be observed, and, among these, a homogeneous epileptic phenotype is possible to recognize. For instance, cortical malformations as the agyria-pachygyria spectrum or nodular heterotopia, mainly associated to mutations in the Lissencephaly 1 (LIS1), Doublecortin (DCX), Filamin A (FLNA) or Reelin (REL) genes, all encoding MTs, each of them with a specific role in causing epilepsy, show a typical epileptic phenotype as far as concern the onset, frequency and type of seizures and response to therapy $[15,29]$. On the contrary, mutations in $\alpha$-and $\beta$-tubulin genes, responsible for a large spectrum of cortical and brain malformations do not show such a high specificity and correlation with the epileptic phenotype.

Among the $\alpha$ - and $\beta$-tubulin genes family, those mainly associated with epilepsy are Tubulin $\alpha-1 \mathrm{~A}$ (TUBA1A) (about $50 \%$ of the cases), Tubulin $\beta-2 B$ (TUBB2B) (about 40\%), and Tubulin $\beta-4 A$ (TUBB4A) (about $40 \%$ ). On the contrary, mutations in Tubulin $\beta-3$ (TUBB3) gene cause epilepsy only in about $15 \%$ of cases, while seizures has never been described in Tubulin $\beta$ (TUBB) gene [7].

\section{Tubulin a-1A (TUBA1A) gene [ORPHA171680, OMIM 611603]}

MCDs associated to mutations in the TUBA1A gene showed various degrees of lissencephaly, ranging from complete loss of gyri and sulci (agyria) to brain with simplified abnormally thick convolutions (pachygyria) and classical lissencephaly.[10,25] More recently, this spectrum of MCDs was broadened and associations with perisylvian PMG/PMG-like patterns have been also documented.[24-26] Histological examinations in brain affected by TUBA1A mutations revealed structural abnormalities of cortex, a fractured pyramidal layer of the hippocampus, defects attributed to impairment of neuronal migration mechanisms. These phenotypes were reminiscent of mouse models of lissencephaly (LIS1, DCX, REL) [30].

The TUBA1A gene is highly expressed in post-mitotic neurons, but not in glia in the human and mouse brain. Murine expression studies showed that the TUBA1A gene is largely absent from the proliferative ventricular zone and its expression peaks at 16.5 embrionic day when neurons migration require extension of leading processes (i.e., traslocation of the nucleus and retraction of trailing processes) [24]. Similarly, neuritis outgrowth requires the stable support and dynamic force generated by microtubules. All these processes are reliant on a dynamic of microtubules networks and they are impaired in mutations in the TUBA1A genes, primarily leading to disorders of migration respect to axonal guidance [30].

The epilepsy described to date in patients carrying mutations in the TUBA1A gene (about $50 \%$ of the cases) present various seizures patterns, including early-onset epileptic seizures as infantile spasms, tonic, early generalized tonic-clonic, and focal seizures [7]. These may evolve in atypical absence seizures or myoclonic and atonic drop seizures in later childhood.

\section{Tubulin $\beta$-2B (TUBB2B) gene [ORPHA300573, OMIM 610031]}

Bilateral asymmetric PMG was the initial phenotype associated with mutations in the TUBB2B gene [30]. Subsequently, the spectrum of associated MCDs has broadened and various types of lissencephaly as well as more complex gyral disorganization and schizencephaly ( $\mathrm{SCH}$ ) were found [31-35]. In a recent study on mice models, Breuss et al analyzed the expression pattern of the tubulin isoform tubb2b, high in postmitotic regions dominated by migrating and differentiating neurons, which is restricted to macroglia in the 8-week-old cortex (corpus callosum, anterior commissure and internal capsule) [36]. Its expression involve hippocampi, Purkyinje and granule cells layer of cerebellum. Analysis conducted on TUBB2B gene mutations to date, concluded that, as for the TUBA1A gene, these mutations influence the tubulin heterodimer folding and their incorporation into microtubules in different ways, thus leading to combinations of impairment in neuronal migration and radial glia dysfunction [31]. Epilepsy was described in about $40 \%$ of subjects carrying TUBB2B gene defects with a wide range of presentation (infantile spasms and focal seizures) $[7,33]$.

\section{Tubulin $\beta-3$ (TUBB3) gene [ORPHA300570, OMIM 614039]}

Mutations in the TUBB3 gene were first described in axon guidance disorders (e.g., corpus callosum abnormalities, hypoplasia of oculomotor nerves and congenital fibrosis of extraocular muscles), $[37,38]$ without neuronal migration defects or microcephaly in contrast with previously described TUBA1A and TUBB2B gene mutations. Thereafter, $\mathrm{PMG}$ and simplified and disorganized gyral patterning were also described in few individuals carrying defects in the TUBB3 gene $[39,40]$. More recently, a specific mutation of the TUBB3 gene (i.e., EK104 phenotype) was described in a new fascinating phenotype as resulting from an axonal path-finding defects characterized by peripheral neuropathy, defective olfactory function, photophobia, cyclic vomiting and hypogonatropic hypogonadism $[29,40]$. Taken together, these findings imply that the phenotype related to the TUBB3 gene involve primarily axonal guidance mechanisms with low levels of epileptogenicity in subjects carrying TUBB3 gene mutations (about 15\% of cases show seizures) [7].

\section{Tubulin $\beta$-4A (TUBB4A) gene [ORPHA98805 OMIM 602662]}

In recent years, a specific phenotype has been described in association with mutations in the TUBB4 gene, characterized by whispering dysphonia, generalized dystonia, ataxic gait (hobby horse), but without neuroradiological and defined dystonia type 4 (DYT4) [41,42]. A rare form of leukoencephalopathy, chacterized by hypomielination with atrophy of the basal ganglia and cerebellum $(\mathrm{H}-$ $\mathrm{ABC}$ ), has been described also associated to mutations of the TUBB4A gene [18-21], supporting the hypothesis that tubulin genes have subtle, but yet distinct roles in microtubular dynamics and functions, according to cell type and neurodevelopmental time points [9]. Despite the underlying cellular and molecular mechanisms responsible for these phenotypes remain to be defined, it has been demonstrated that this gene is expressed at low levels in developing brain, but it is highly transcripted in adult cerebellum, brainstem and striatum [30]. Intriguingly, also in one patient carrying mutation in the TUBB3 without supratentorial malformation - but only with cerebellar dysplasia - partial complex seizure are described. Epilepsy is present in about $40 \%$ of the subjects carrying mutations in the TUBB4A gene [7,22].

For the following tubulin genes the number of mutated patients is limited and observations are difficult to draw:

\section{Tubulin a-8 (TUBA8) [ORPHA2509, OMIM 613180]}

Only one mutation was reported of the TUBA8A gene, observed in two related families associated to generalized PMG and optic nerve 
hypoplasia [43]. Braun et al showed in their study, the expression at very low levels of the TUBA8 gene in the developing brain in mice and humans suggesting its role is not pathogenic at all [24]. All the cases described showed seizures [7].

\section{Tubulin, $\beta$-2A (TUBB2A) gene [OMIM 615101]}

Mutations in the TUBB2 gene were described in two unrelated individuals with simplified gyral patterning and infantile-onset focal epilepsy in all the cases [17].

\section{Tubulin $\beta$ (TUBB) gene [OMIM 191130]}

Mutations in the TUBB gene have been described in three unrelated cases, all presenting microcephaly, one of them showing a phenotype characterized by focal PMG associated with localized band heterotopias, none showing epilepsy [39]. Studies on transgenic mouse showed that the TUBB gene is expressed in radial glial progenitors, intermediate progenitors and post-mitotic neurons, so it is involved in neurogenic division and migration processes and its depletion result in neuronal migration defects. It not clear why it is not associated with epileptic seizures, but surely the use of larger samples of patients carrying TUBB gene mutations is warranted in future studies, in order to achieve a better comprehension of the associated disease and clinical patterns.

\section{Conclusion}

The present review confirms the involvement of mutations in the tubulin-encoding genes in a large spectrum of brain malformations, and their role in epilepsy determinism.

Epileptogenicity of tubulin genes' mutations is a complex phenomenon, depending on the way they alter the dynamic properties and functions of microtubules. Seizures appear mainly when mutations lead to MCDs as it happens in TUBA1A, TUBB2B and TUBB3, while they are less frequent when mutations are associated with axonal guidance disorders.

Epileptic seizures, when present, start early (i.e., during the first year of life) and are more often generalized (e.g., infantile spasms, tonic, tonic-clonic) as expression of a diffuse cortical maldevelopment. A specific pattern is not described and severity show a large range of variability

Little is known about outcome even if drug resistant seizures are very rare and a good response to therapy is often observed.

\section{Funding}

This study was partially supported by Italian Ministry of Health grants to RB (RC/01/03/2011) and Institutional funds to RB (5x Mille)

\section{References}

1. Nabbout R, Scheffer IE (2013) Genetics of idiopathic epilepsies. Handb Clin Neurol 111: 567-578.

2. Barkovich AJ, Guerrini R, Kuzniecky RI, Jackson GD, Dobyns WB (2012) A developmental and genetic classification for malformations of cortical development: update 2012. Brain 135: 1348-1369.

3. Andrade DM (2009) Genetic basis in epilepsies caused by malformations of cortical development and in those with structurally normal brain. Hum Genet 126: 173-193.
4. Guerrini R, Dobyns WB (2014) Malformations of cortical development: clinical features and genetic causes. Lancet Neurol 13: 710-726.

5. Kuzniecky R (2015) Epilepsy and malformations of cortical development: new developments. Curr Opin Neurol 28: 151-157.

6. Wu Q, Liu J, Fang A, Li R, Bai Y, et al. (2014) The dynamics of neuronal migration. Adv Exp Med Biol 800: 25-36.

7. Romaniello R, Arrigoni F, Bassi MT, Borgatti R (2015) Mutations in Îtand $\hat{\mathrm{I}}^{2}$-tubulin encoding genes: implications in brain malformations. Brain Dev 37: 273-280.

8. Guerrini R, Barba C (2010) Malformations of cortical development and aberrant cortical networks: epileptogenesis and functional organization. J Clin Neurophysiol 27: 372-379.

9. Tischfield MA, Engle EC (2010) Distinct alpha- and beta-tubulin isotypes are required for the positioning, differentiation and survival of neurons: new support for the 'multi-tubulin' hypothesis. Biosci Rep 30: 319-330.

10. Kumar RA, Pilz DT, Babatz TD, Cushion TD, Harvey K, et al (2010) TUBA1A mutations cause wide spectrum lissencephaly (smooth brain) and suggest that multiple neuronal migration pathways converge on alpha tubulins. Hum Mol Genet 19: 2817-2827.

11. Jaglin XH, Chelly J (2009) Tubulin-related cortical dysgeneses: microtubule dysfunction underlying neuronal migration defects. Trends Genet 25: 555-566.

12. Singh KK, Tsai LH (2010) MicroTUB(B3)ules and brain development. Cell 140: 30-32.

13. Tischfield MA, Cederquist GY, Gupta ML, Engle EC (2011) Phenotypic spectrum of the tubulin-related disorders and functional implications of disease-causing mutations. Curr Opin Genet Dev 21: 286-294.

14. Qu C, Dwyer T, Shao Q, Yang T, Huang H, et al. (2013) Direct binding of TUBB3 with DCC couples netrin-1 signaling to intracellular microtubule dynamics in axon outgrowth and guidance. J Cell Sci 126: 3070-3081.

15. Cushion TD, Dobyns WB, Mullins JG, Stoodley N, Chung SK, et al. (2013) Overlapping cortical malformations and mutations in TUBB2B and TUBA1A. Brain 136: 536-548.

16. Poirier K, Saillour Y, Bahi-Buisson N, Jaglin XH, Fallet-Bianco C, et al. (2010) Mutations in the neuronal B-tubulin subunit TUBB3 result in malformation of cortical development and neuronal migration defects. Hum Mol Genet 19: 4462-4473.

17. Cushion TD, Paciorkowski AR, Pilz DT, Mullins JG, Seltzer LE, et al. (2014) De novo mutations in the beta-tubulin gene TUBB2A cause simplified gyral patterning and infantile-onset epilepsy. Am J Hum Genet 94: 634-641.

18. Blumkin L, Halevy A, Ben-Ami-Raichman D, Dahari D, Haviv A, et al. (2014) Expansion of the spectrum of TUBB4A-related disorders: a new phenotype associated with a novel mutation in the TUBB4A gene. Neurogenetics 15: 107-113.

19. Ferreira C, Poretti A, Cohen J, Hamosh A, Naidu S (2014) Novel TUBB4A mutations and expansion of the neuroimaging phenotype of hypomyelination with atrophy of the basal ganglia and cerebellum $(\mathrm{H}-$ ABC). Am J Med Genet A 164A: 1802-1807.

20. Purnell SM, Bleyl SB, Bonkowsky JL (2014) Clinical exome sequencing identifies a novel TUBB4A mutation in a child with static hypomyelinating leukodystrophy. Pediatr Neurol 50: 608-611.

21. Hamilton EM, Polder E, Vanderver A, Naidu S, Schiffmann R, et al. (2014) Hypomyelination with atrophy of the basal ganglia and cerebellum: further delineation of the phenotype and genotypephenotype correlation. Brain 137: 1921-1930.

22. Oegema R, Cushion TD, Phelps IG, Chung SK, Dempsey JC, et al. (2015) Recognizable cerebellar dysplasia associated with mutations in multiple tubulin genes. Hum Mol Genet 24: 5313-5325.

23. Romaniello R, Tonelli A, Arrigoni F, Baschirotto C, Triulzi F, et al. (2012) A novel mutation in the $\hat{\mathrm{I}}^{2}$-tubulin gene TUBB2B associated with complex malformation of cortical development and deficits in axonal guidance. Dev Med Child Neurol 54: 765-769. 
24. Braun A, Breuss M, Salzer MC, Flint J, Cowan NJ, et al. (2010) Tuba8 is expressed at low levels in the developing mouse and human brain. Am J Hum Genet 86: 819-822.

25. Poirier K, Keays DA, Francis F, Saillour Y, Bahi N, et al. (2007) Large spectrum of lissencephaly and pachygyria phenotypes resulting from de novo missense mutations in tubulin alpha 1A (TUBA1A). Hum Mutat 28 1055-1064.

26. Bahi-Buisson N, Poirier K, Boddaert N, Saillour Y, Castelnau L, et al. (2008) Refinement of cortical dysgeneses spectrum associated with TUBA1A mutations. J Med Genet 45: 647-653.

27. Bahi-Buisson N, Poirier K, Fourniol F, Saillour Y, Valence S, et al. (2014) The wide spectrum of tubulinopathies: what are the key features for the diagnosis? Brain 137: 1676-1700.

28. Leventer RJ, Guerrini R, Dobyns WB (2008) Malformations of cortical development and epilepsy. Dialogues Clin Neurosci 10: 47-62.

29. Barkovich J (2013) Complication begets clarification in classification Brain 136: 368-373.

30. Breuss M, Keays DA (2014) Microtubules and neurodevelopmental disease: the movers and the makers. Adv Exp Med Biol 800: 75-96.

31. Jaglin XH, Poirier K, Saillour Y, Buhler E, Tian G, et al. (2009) Mutations in the beta-tubulin gene TUBB2B result in asymmetrical polymicrogyria. Nat Genet 41: 746-752.

32. Cederquist GY, Luchniak A, Tischfield MA, Peeva M, Song Y, et al. (2012) An inherited TUBB2B mutation alters a kinesin-binding site and causes polymicrogyria, CFEOM and axon dysinnervation. Hum Mol Genet 21: 5484-5499.

33. Guerrini R, Mei D, Cordelli DM, Pucatti D, Franzoni E, et al. (2012) Symmetric polymicrogyria and pachygyria associated with TUBB2B gene mutations. Eur J Hum Genet 20: 995-998.

34. Romaniello R, Arrigoni F, Cavallini A, Tenderini E, Baschirotto C, et al (2014) Brain malformations and mutations in $\hat{I} \pm-$ and $\hat{I}^{2}$-tubulin genes: a review of the literature and description of two new cases. Dev Med Child Neurol 56: 354-360.

35. Uribe V (2010) The beta-tubulin gene TUBB2B is involved in a large spectrum of neuronal migration disorders. Clin Genet 77: 34-35.

36. Breuss M, Morandell J, Nimpf S, Gstrein T, Lauwers M, et al. (2015) The Expression of Tubb2b Undergoes a Developmental Transition in Murine Cortical Neurons. J Comp Neurol 523: 2161-2186.

37. Tischfield MA, Baris HN, Wu C, Rudolph G, Van Maldergem L, et al. (2010) Human TUBB3 mutations perturb microtubule dynamics, kinesin interactions, and axon guidance. Cell 140: 74-87.

38. Saillour Y, Broix L, Bruel-Jungerman E, Lebrun N, Muraca G, et al (2014) Beta tubulin isoforms are not interchangeable for rescuing impaired radial migration due to Tubb3 knockdown. Hum Mol Genet 23: 1516-1526.

39. Breuss M, Heng JI, Poirier K, Tian G, Jaglin XH, et al. (2012) Mutations in the $\hat{I}^{2}$-tubulin gene TUBB5 cause microcephaly with structural brain abnormalities. Cell Rep 2: 1554-1562.

40. Chew S, Balasubramanian R, Chan WM, Kang PB, Andrews C, et al. (2013) A novel syndrome caused by the E410K amino acid substitution in the neuronal $\hat{\mathrm{I}}^{2}$-tubulin isotype 3. Brain 136: 522-535.

41. Hersheson J, Mencacci NE, Davis M, MacDonald N, Trabzuni D, et al. (2013) Mutations in the autoregulatory domain of $\hat{\mathrm{I}}^{2}$-tubulin 4a cause hereditary dystonia. Ann Neurol 73: 546-553.

42. Lohmann K, Wilcox RA, Winkler S, Ramirez A, Rakovic A, et al. (2013) Whispering dysphonia (DYT4 dystonia) is caused by a mutation in the TUBB4 gene. Ann Neurol 73: 537-545.

43. Abdollahi MR, Morrison E, Sirey T, Molnar Z, Hayward BE, et al. (2009) Mutation of the variant alpha-tubulin TUBA8 results in polymicrogyria with optic nerve hypoplasia. Am J Hum Genet 85: 737-744. 